\title{
Acute Cerebral Hemodynamic Insufficiency after Plaque Haemorrhage
}

\author{
Leodante da Costa, Marcelo Cunha, Nir Lipsman
}

Keywords: Carotid artery disease, Stroke imaging

doi:10.1017/cjn.2016.252

Can J Neurol Sci. 2016; 43: 717-718

Hemodynamic insufficiency in association with severe unilateral occlusive carotid disease (OCD) is a rare cause of stroke. Most cases of stroke due to hemodynamic insufficiency are related to low-output cardiac states and hypotension. Carotid intraplaque haemorrhage is a known complicating factor of OCD and has been associated with higher rates of thromboembolic ischaemic events. We present a case of documented acute symptomatic left middle cerebral artery (MCA) territory perfusion deficit without intracranial atherosclerosis/stenosis/thrombus and associated with plaque haemorrhage, completely reversed by carotid angioplasty and stenting.

A 70-year-old male was admitted with sudden onset of right-hand weakness and aphasia lasting for about an hour. He had improved completely by the time of arrival. Medical history was negative for diabetes and hyperlipidemia. He denied a history but presented to the ER with very high blood pressure $(220 / 120 \mathrm{mmHg})$. Attempts to lower it resulted in repeated deficits.

Head computerized tomography (CT) showed an early small stroke in the left fronto-parietal region, suggesting a watershed infarct (Figure 1A: arrowheads). CT angiography (CTA) showed severe (measured at $77 \%$ by NASCET criteria) left cervical carotid stenosis (Figure 1B), mild right cervical carotid stenosis at the bifurcation and no evidence of intracranial stenosis or clot (Figure 1C). CT perfusion showed an increased mean transit time (MTT), decreased blood flow (CBF) and normal blood volume (CBV) in the left MCA territory (Figure 1E). MRI with MRA angiography (MRA) of the brain and craniocervical vessels confirmed the small ischaemic areas in the high left frontal and parietal regions without other areas of restricted diffusion. It also showed evidence of acute haemorrhage in the left carotid plaque (Figure 1D).

An urgent carotid angioplasty and stenting were performed approximately 10 hours after the initial presentation, with good radiological results (Figure 1F). A CT/CTA/CTP less than 6 hours after this procedure showed total resolution of the perfusion mismatch (Figure 1G).
Thromboembolic events related to carotid atherosclerotic disease are the underlying cause of a large percentage of strokes. ${ }^{1}$ Intraplaque haemorrhage (IPH) has been described as a risk factor for recurrent emboli and stroke $\mathrm{e}^{2-7}$ due to recurrent thromboembolic phenomena. ${ }^{8,9}$ Hemodynamic cerebral events may result from low cardiac output (orthostatic ischaemia, myocardial ischemia, cardiac arrhythmias) or severe carotid stenosis or occlusion, and they have been implicated as the underlying mechanism in roughly $10 \%$ of all strokes. ${ }^{10}$ Approximately two-thirds of cases are associated with severe carotid stenosis, often bilateral, or carotid occlusion.

In the absence of any evidence of intracranial thrombus and a large area of mismatch in the MCA territory, we speculate that plaque rupture and haemorrhage lead to acute hemodynamic insufficiency. ${ }^{11,12}$ The likely compensatory increase in blood pressure and the recurrent deficits with attempts to lower it support this hypothesis. Also, the patient had a carotid ultrasound at another institution two weeks before, due to arm weakness, that showed no hemodynamically significant carotid stenosis. A computerized tomography done at the same time also showed no stroke.

Another possibility was that a clot occluding the MCA resolved rapidly enough not to be picked up in the first imaging. This is unlikely due to the short time elapsed between the beginning of his symptoms and the CT scan (40 minutes to conclusion of the scan), and it would not explain the complete resolution of his recurrent symptoms and perfusion mismatch after carotid revascularization.

This case illustrates what seems to be a rare association of intraplaque haemorrhage and acute hemodynamic insufficiency in the absence of contralateral craniocervical vascular occlusive disease oripsilateral intracranial occlusive disease.

\section{DisCLOSURES}

Leodante da Costa, Marcelo Cunha and Nir Lipsman hereby declare that they have nothing to disclose.

\footnotetext{
From the Division of Neurosurgery, Sunnybrook Hospital, University of Toronto, Toronto, Ontario, Canada (LdC, NL); the Department of Neurosurgery, Hospital Mater Dei, Belo Horizonte, Minas Gerais, Brazil (MC).

Received August 10, 2014. Final Revisions Submitted November 12, 2015. Date of Acceptance January 8, 2016

Correspondence to: Leodante da Costa, Sunnybrook Health Sciences Centre, 2075 Bayview Avenue, Room A1 37, Toronto, Ontario M4N 3M, Canada. Email: leo.dacosta@ sunnybrook.ca
} 


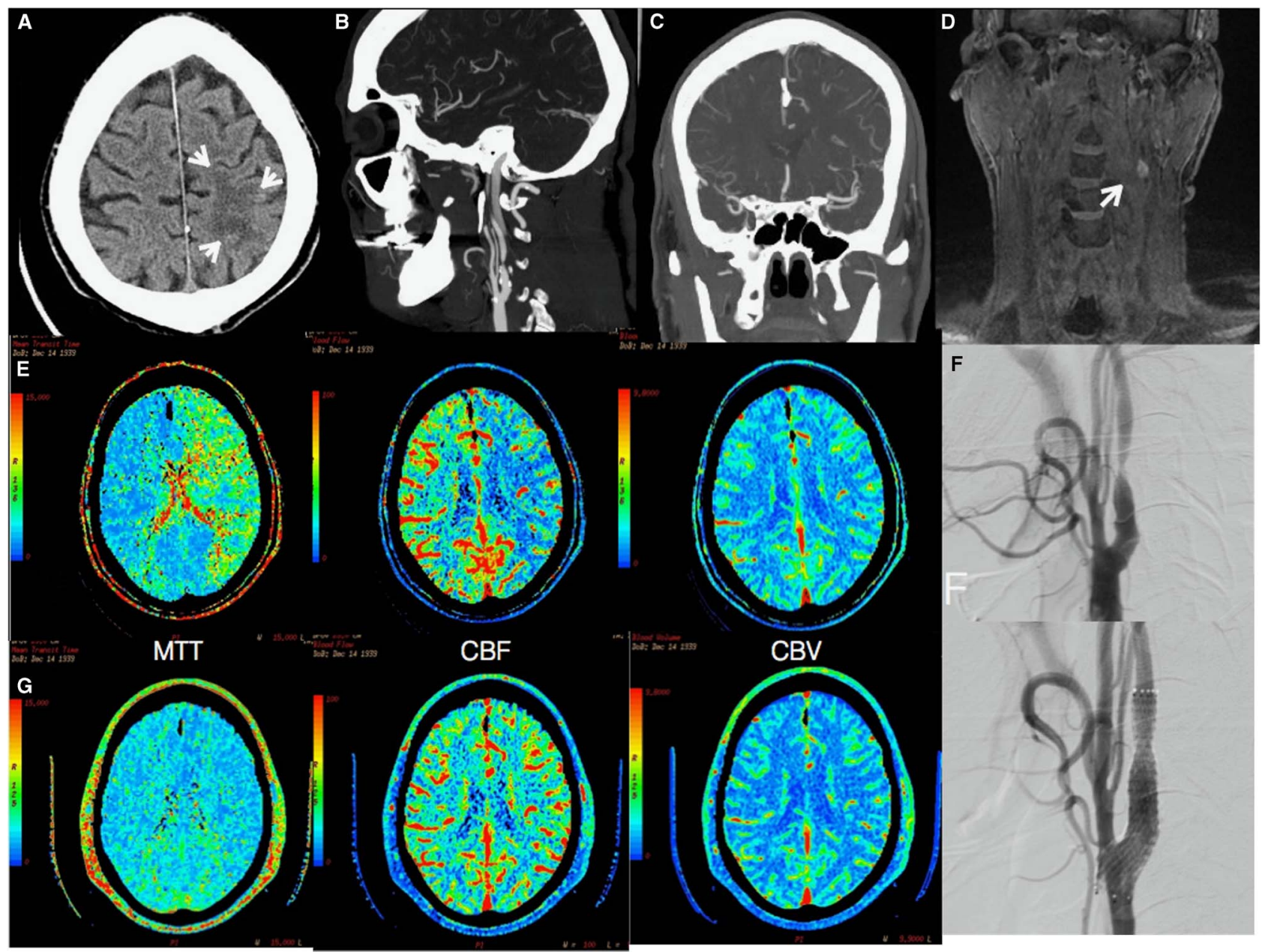

Figure 1: Plain CT head showing a small stroke in the left fronto-parietal region, suggesting an watershed infarct (A - arrowheads). CT angiogram: severe left cervical carotid stenosis $(B)$, and no evidence of intracranial stenosis or clot $(C)$. CT perfusion with an increased mean transit time (MTT), decreased blood flow $(C B F)$ and normal blood volume $(C B V)$ in the left MCA territory $(E)$. Acute hemorrhage in left carotid plaque $(D)$ on MRI. Carotid angioplasty and stenting $(F)$ followed by improvement of CTP parameters and total resolution of the perfusion mismatch $(G)$.

\section{REFERENCES}

1. Lanzino G, Tallarita T, Rabinstein AA. Internal carotid artery stenosis: natural history and management. Semin Neurol. 2010; 30(5):518-27. Epub after print Jan 4, 2011.

2. Altaf N, Beech A, Goode SD, Gladman JR, Moody AR, Auer DP, et al. Carotid intraplaque hemorrhage detected by magnetic resonance imaging predicts embolization during carotid endarterectomy. J Vasc Surg. 2007;46(1):31-6. Epub ahead of print Jun 1.

3. Altaf N, MacSweeney ST, Gladman J, Auer DP. Carotid intraplaque hemorrhage predicts recurrent symptoms in patients with highgrade carotid stenosis. Stroke. 2007;38(5):1633-5. Epub ahead of print Mar 22.

4. Gao P, Chen ZQ, Bao YH, Jiao LQ, Ling F. Correlation between carotid intraplaque hemorrhage and clinical symptoms: systematic review of observational studies. Stroke. 2007;38(8):2382-90. Epub ahead of print Jun 28.

5. Gao P, Chen ZQ, Jiao LQ, Ling F. The correlation of carotid plaque pathohistologic features and neurological symptoms: a metaanalysis of observational studies. Neurol India. 2007;55(2):122-9.

6. Altaf N, Daniels L, Morgan PS, Auer D, MacSweeney ST, Moody $\mathrm{AR}$, et al. Detection of intraplaque hemorrhage by magnetic resonance imaging in symptomatic patients with mild to moderate carotid stenosis predicts recurrent neurological events. J Vasc Surg. 2008;47(2):337-42. doi: 10.1016/j.jvs.2007.09.064.
7. Hosseini AA, Kandiyil N, MacSweeney ST, Altaf N, Auer DP. Carotid plaque hemorrhage on magnetic resonance imaging strongly predicts recurrent ischemia and stroke. Ann Neurol. 2013;73(6):774-84. Epub ahead of print Jun 4.

8. Spagnoli LG, Mauriello A, Sangiorgi G, Fratoni S, Bonanno E, Schwartz RS, Piepgras DG, et al. Extracranial thrombotically active carotid plaque as a risk factor for ischemic stroke. JAMA. 2004;292(15):1845-52. PMID: 15494582.

9. Redgrave JN, Lovett JK, Gallagher PJ, Rothwell PM. Histological assessment of 526 symptomatic carotid plaques in relation to the nature and timing of ischemic symptoms: the Oxford plaque study. Circulation. 2006;113(19):2320-8. Epub ahead of print May 1.

10. Bladin CF, Chambers BR. Frequency and pathogenesis of hemodynamic stroke. Stroke. 1994;25(11):2179-82. PMID: 7974542.

11. Derdeyn CP, Grubb RL Jr., Powers WJ. Cerebral hemodynamic impairment: methods of measurement and association with stroke risk. Neurology. 1999;53(2):251-9. Epub after print Aug 3.

12. Nemoto EM, Yonas H, Kuwabara H, Pindzola RR, Sashin D, Meltzer CC, et al. Identification of hemodynamic compromise by cerebrovascular reserve and oxygen extraction fraction in occlusive vascular disease. J Cereb Blood Flow Metab. 2004; 24(10):1081-9. PMID: 15529008. 